\title{
Isospectral circular membranes
}

\author{
H P W Gottlieb
}

School of Science, Griffith University, Nathan, Queensland 4111, Australia

E-mail: H.Gottlieb@griffith.edu.au

17 October 2003

PACS classification numbers: 02.30.Jr, 02.30.Zz, 43.40.Dx

\begin{abstract}
A prescription for finding the densities of circular membranes which are isospectral to a uniform circular membrane is extended to cover isospectrality to any circular membrane density, thereby also revealing the underlying grouptheoretical structure which is further elucidated. Membranes isospectral to radial-density membranes no longer possess the radial symmetry, and some examples are discussed.
\end{abstract}




\section{Introduction}

In a previous paper [1], the author used a particular conformal map to find the densities of inhomogeneous circular membranes which were isospectral to a homogeneous circular membrane. In this paper, the method is extended to enable one to construct densities for circular membranes which are isospectral to any given circular membrane density, for fixed (Dirichlet) and free (Neumann) boundary conditions. The formalism is also embedded in a group-theoretical structure, which illuminates further features. Examples of non-radially-symmetric densities for membranes isospectral to radial-density membranes are discussed.

As well as providing interesting classes of complicated density functions for circular membranes whose spectra are obtainable from simpler cases, this work provides further information for inverse problems in two dimensions by exemplifying density non-uniqueness for a given spectrum for a fixed shape.

The partial differential equation for the small transverse vibrations $\mathrm{u}(\mathrm{x}, \mathrm{y})$ of a membrane having density $\sigma(x, y)$, unit tension, and characteristic angular frequencies $\omega$ is

$$
\nabla^{2} u \equiv \frac{\partial^{2} u}{\partial x^{2}}+\frac{\partial^{2} u}{\partial y^{2}}=-\omega^{2} \sigma(x, y) u(x, y)
$$

For circular membranes of radius $\mathrm{C}$, the standard boundary conditions are

or

$$
\mathrm{u}=0
$$

$$
\frac{\partial u}{\partial r}=0
$$

on $r \equiv \sqrt{x^{2}+y^{2}}=C$ for fixed or free rim respectively.

\section{Transformations}

Let $\mathrm{z}_{\mathrm{j}}=\mathrm{x}_{\mathrm{j}}+\mathrm{i} \mathrm{y}_{\mathrm{j}}(\mathrm{j}=1,2)$ be a pair of complex variables in two coordinate systems, related by

$$
\mathrm{z}_{1}=\mathrm{f}_{1}\left(\mathrm{z}_{2}\right)
$$

where $f_{1}(z)$ is a one-to-one analytic function of $z$. Then, as a consequence of the CauchyRiemann equations [c.f. 2],

$$
\nabla_{2}^{2} \mathrm{~F}_{2}\left(\mathrm{x}_{2}, \mathrm{y}_{2}\right)=\left|\mathrm{f}_{1}\left(\mathrm{z}_{2}\right)\right|^{2} \nabla_{1}^{2} \mathrm{~F}_{1}\left(\mathrm{x}_{1}, \mathrm{y}_{1}\right)
$$


where $\mathrm{F}_{1}\left(\mathrm{x}_{1}, \mathrm{y}_{1}\right)=\mathrm{F}_{2}\left(\mathrm{x}_{2}, \mathrm{y}_{2}\right)$ are functions of the coordinates (and the Laplacian subscripts denote the coordinate subscripts in the partial derivatives appearing therein).

Furthermore, if the normal derivative of $F_{2}$ along a curve in $z_{2}$-plane is zero, then the normal derivative of $F_{1}$ along the image curve in the $\mathrm{z}_{1}$-plane is also zero. (Obviously, also $F_{1}$ itself is zero iff $F_{2}$ is zero.)

Let $\mathrm{u}_{1}\left(\mathrm{x}_{1}, \mathrm{y}_{1}\right)$ be the vibration displacement of a membrane and $\mathrm{u}_{2}\left(\mathrm{x}_{2}, \mathrm{y}_{2}\right)$ that for a second membrane, related through (2.1) by

$$
\mathrm{u}_{1}\left(\mathrm{x}_{1}, \mathrm{y}_{1}\right)=\mathrm{u}_{2}\left(\mathrm{x}_{2}, \mathrm{y}_{2}\right)=\mathrm{u}_{1}\left(\mathrm{x}_{1}\left(\mathrm{x}_{2}, \mathrm{y}_{2}\right), \mathrm{y}_{1}\left(\mathrm{x}_{2}, \mathrm{y}_{2}\right)\right)
$$

By (2.2), and (1.1) for $\left(\mathrm{x}_{1}, \mathrm{y}_{1}\right)$,

$$
\nabla_{2}^{2} u_{2}\left(x_{2}, y_{2}\right)=-\omega^{2}\left|f_{1}^{\prime}\left(z_{2}\right)\right|^{2} \sigma_{1}\left(x_{1}, y_{1}\right) u_{2}
$$

This is the eigenvalue equation for a vibrating membrane with density

$$
\sigma_{2}\left(x_{2}, y_{2}\right)=\left|f_{1}^{\prime}\left(z_{2}\right)\right|^{2} \sigma_{1}\left(x_{1}\left(x_{2}, y_{2}\right), y_{1}\left(x_{2}, y_{2}\right)\right)
$$

which is isospectral with the membrane with density $\sigma_{1}\left(\mathrm{x}_{1}, \mathrm{y}_{1}\right)$.

With a view to obtaining isospectral circular membranes, it is now recalled that the only one-to-one analytic mappings of a circular disc of radius $\mathrm{C}$ onto itself (up to simple rotations in the complex planes) is given by [2]

$$
z_{1}=f_{1}\left(z_{2}\right)=\frac{z_{2}-a_{1} C}{1-\left(\frac{\left.a_{1} / C\right) z_{2}}{C}\right.}, \quad-1<a_{1}<1 .
$$

Moreover, as mentioned after (2.2) above, such a transformation does preserve the Dirichlet and Neumann boundary conditions on the rims since $\left|z_{1}\right|=C$ maps onto $\left|z_{2}\right|=C$. Specifically,

$$
\begin{aligned}
& x_{1}=\frac{\left(x_{2}-a_{1} C\right)\left(1-\left(\frac{a_{1}}{C}\right) x_{2}\right)-\left(\frac{a_{1}}{C}\right) y_{2}^{2}}{D_{a_{1}}\left(x_{2}, y_{2}\right)}, \\
& y_{1}=\frac{\left(1-a_{1}^{2}\right) y_{2}}{D_{a_{1}}\left(x_{2}, y_{2}\right)}
\end{aligned}
$$

where 


$$
D_{a}(x, y)=\left(1-\left(\frac{a}{c}\right) x\right)^{2}+\frac{a^{2}}{c^{2}} y^{2}
$$

For any circular membrane density $\sigma_{1}\left(\mathrm{x}_{1}, \mathrm{y}_{1}\right)$, there is therefore a family of isospectral circular membranes with the same boundary condition (Dirichlet or Neumann) with densities given from (2.5) with (2.6) and (2.7) by

$$
\sigma_{2}\left(x_{2}, y_{2}\right)=\frac{\left(1-a_{1}^{2}\right)^{2}}{\left[D_{a_{1}}\left(x_{2}, y_{2}\right)\right]^{2}} \sigma_{1}\left(x_{1}\left(x_{2}, y_{2}\right), y_{1}\left(x_{2}, y_{2}\right)\right),-1<a_{1}<1
$$

(For a given $\mathrm{a}_{1}$, replacement by $-\mathrm{a}_{1}$ corresponds to changing the sign of $\mathrm{x}$-coordinates, i.e. "flipping" the discs about the $y$-axis; but all other $a_{1}$ values yield physically distinct membranes.)

The corresponding characteristic functions are given by $\mathrm{u}_{2}\left(\mathrm{x}_{2}, \mathrm{y}_{2}\right)$ as in (2.3) with (2.7).

\section{Group-theoretical structure}

The presentation may be cast in the formalism of a Lie transformation group development, along the lines of [3] (which dealt with the one-dimensional problem of isospectral strings), to bring out the mathematical structure more clearly.

First of all, the identity transformation $\mathrm{z}_{1}=\mathrm{z}_{2}$ obviously corresponds to parameter $\mathrm{a}=0$. The inverse of the transformation (2.6) is given by

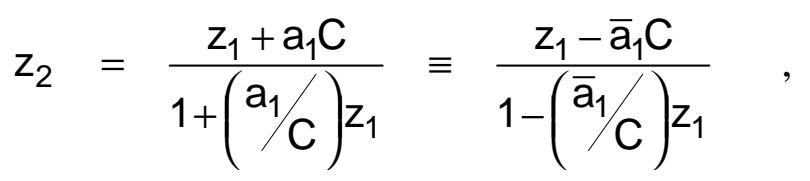

i.e. of the same form as (2.6). Thus, corresponding to a coordinate transformation with parameter $\mathrm{a}$, the inverse transformation has parameter given by

$$
\overline{\mathrm{a}}=-\mathrm{a}
$$

(This is unrelated to the observation above that a transformation from $\mathrm{z}_{1}$ to $\mathrm{z}_{2}$ with parameter -a yields a membrane density physically equivalent (via change of $x-$ coordinate signs) to a transformation still from $\mathrm{z}_{1}$ to $\mathrm{z}_{2}$ with parameter $+\mathrm{a}$.)

As a matter of fact [2], for conformal transformations of complex variables, the derivative of a transformation is the reciprocal of the derivative of the inverse transformation. With reference to (2.4) and (2.5), this shows that not only are all eigenfrequencies for $\sigma_{1}$ eigenfrequencies for $\sigma_{2}$, but also all eigenfrequencies for $\sigma_{2}$ are eigenfrequencies for $\sigma_{1}$, so there truly is complete isospectrality. 
Next, consider successive transformations corresponding to (2.6), (2.7) with, further,

$$
z_{2}=f_{2}\left(z_{3}\right)=\frac{z_{3}-a_{2} C}{1-\left(a_{2} / c\right) z_{3}} \quad, \quad-1<a_{2}<1,
$$

with $\mathrm{x}_{3}$ and $\mathrm{y}_{3}$ corresponding to $\mathrm{z}_{3}$. Then it can be shown that

$$
z_{1}=\frac{z_{3}-a_{1,2} C}{1-\left(a_{1,2} / c\right) z_{3}}
$$

where

$$
a_{1,2}=\frac{a_{1}+a_{2}}{1+a_{1} a_{2}}
$$

This proves closure of the coordinate transformations, and is the parameter composition law. (The inverse transformation parameter, for which, $a_{1,2}=0$, is confirmed to be $a_{2}=-$ $\mathrm{a}_{1}$, as in (3.2) above.)

Moreover, successive transformations with expressions such as (2.5) and (3.3) show that for the densities

$$
\sigma_{3}\left(x_{3}, y_{3}\right)=\left|f_{2}^{\prime}\left(z_{3}\right)\right|^{2} \sigma_{2}\left(x_{2}, y_{2}\right)=\left|f_{2}^{\prime}\left(z_{3}\right)\right|^{2}\left|f_{1}^{\prime}\left(z_{2}\right)\right|^{2} \sigma_{1}\left(x_{1}, y_{1}\right) .
$$

Since the chain rule holds for conformal maps [2], this may be written as

$$
\sigma_{3}\left(x_{3}, y_{3}\right)=\left|f_{1}^{\prime}\left(z_{3}\right)\right|^{2} \sigma_{1}\left(x_{1}, y_{1}\right)
$$

again confirming closure in general for the successive density functions. Specifically for our maps of the form (3.1),

$$
f^{\prime}(z)=\frac{1-a^{2}}{\left(1-\frac{a}{C} z\right)^{2}}
$$

(which leads via (2.5) to (2.8)). Then the equalities inherent in equations (3.6), (3.7), or even (3.6), (2.8), may be verified using the identity

$$
\left(1-a_{1}^{2}\right)\left(1-a_{2}^{2}\right)=\left(1+a_{1} a_{2}\right)^{2}\left(1-a_{1,2}^{2}\right) .
$$


Associativity for the parameters may be verified:

$$
a_{(1,2), 3}=a_{1,(2,3)}=\frac{a_{1}+a_{2}+a_{3}+a_{1} a_{2} a_{3}}{1+a_{1} a_{2}+a_{2} a_{3}+a_{3} a_{1}}
$$

(For the transformations, associativity is guaranteed by the nature of coordinate transformations.) Together with the continuity of the parameters $a_{j}$ and the differentiabilty of the expression (3.5), the above paragraphs verify the Lie group structure.

Equation (3.5) implies that $\mathrm{a}_{1,2}=\mathrm{a}_{2,1}$ : this one-parameter group is, of course, Abelian. The corresponding additive group parameter may be found as in [4, p.294], but is here more easily deduced and verified from the form of (3.5) by comparing with the standard compound-argument formula for $\tanh \left(\alpha_{1}+\alpha_{2}\right)=\left[\tanh \alpha_{1}+\tanh \alpha_{2}\right] /[1+$ $\tanh \alpha_{1} \tanh \alpha_{2}$ ] to be

$$
\alpha=\operatorname{arctanh}(\mathrm{a})
$$

An application of this is to confirm that, for (3.5), $\left|\mathrm{a}_{\mathrm{i}}\right|<1$ implies $\left|\mathrm{a}_{1,2}\right|<1$ as required.

In [3], the author found the infinitesimal generator for coordinate transformations (of a very different kind) for isospectral strings. The infinitesimal generator for transformations (2.8) of the densities in the present paper, corresponding to infinitesimal parameter $\delta \mathrm{a}$, is defined by $\sigma_{2}(\mathrm{x}, \mathrm{y})=[1+\mathrm{i} \delta \mathrm{aQ} \sigma] \sigma_{1}(\mathrm{x}, \mathrm{y})+\mathrm{O}(\delta \mathrm{a})^{2}$, and is found to be

$$
Q_{\sigma}=-i\left[\left(\frac{x^{2}-y^{2}}{C}-C\right) \frac{\partial}{\partial x}+2 \frac{x y}{C} \frac{\partial}{\partial y}+4 \frac{x}{C}\right] \text {. }
$$

\section{Self-isospectral densities and their non-physicality}

It is interesting to know whether there are any density functions which, under a nontrivial transformation of the above type, actually remain unchanged. The action of the generator (3.12) on such an invariant density $\mathrm{g}(\mathrm{x}, \mathrm{y})$ would yield zero: $\mathrm{Q}_{\sigma} \mathrm{g}=0$, i.e. one has to solve the partial differential equation (p.d.e.) for this two-dimensional problem (as against the ordinary differential equation in [3] for the one-dimensional problem there):

$$
\left(x^{2}-y^{2}-C^{2}\right) \frac{\partial g}{\partial x}+2 x y \frac{\partial g}{\partial y}+4 x g(x, y)=0
$$

By following the method of characteristics (e.g. [5, sect. 25.6.1]) one finds that

$$
g(x, y)=\frac{\Gamma(x, y)}{y^{2}}
$$


where $\Gamma(\mathrm{x}, \mathrm{y})$ is constant along the characteristics given by the solutions to the ordinary differential equation

$$
\frac{d y}{d x}=\frac{2 x y}{x^{2}-y^{2}-c^{2}} \text {. }
$$

It may be verified that

$$
\frac{C}{y}-\frac{x^{2}}{C y}-\frac{y}{C}=\text { constant }
$$

is the general solution to (4.3). Thus

$$
g(x, y)=\frac{1}{y^{2}} \Gamma\left(\frac{c^{2}-x^{2}-y^{2}}{C y}\right)
$$

for arbitrary function $\Gamma$, is the general solution to (4.1), yielding the invariant densities.

It may be noted that

$$
\Xi(x, y) \equiv \frac{C^{2}-x^{2}-y^{2}}{C y}
$$

whilst itself not an invariant density, is an invariant function:

$$
\Xi\left(x_{1}\left(x_{2}, y_{2}\right), y_{1}\left(x_{2}, y_{2}\right)\right)=\Xi\left(x_{2}, y_{2}\right)
$$

This may be verified after using (2.7) to first show that

$$
\left[\mathrm{D}_{\mathrm{a}_{1}}\left(\mathrm{x}_{2}, \mathrm{y}_{2}\right)\right]^{2}\left(\mathrm{C}^{2}-\mathrm{r}_{1}^{2}\right)=\left(1-\mathrm{a}_{1}^{2}\right) \mathrm{D}_{\mathrm{a}_{1}}\left(\mathrm{x}_{2}, \mathrm{y}_{2}\right)\left(\mathrm{C}^{2}-\mathrm{r}_{2}^{2}\right)
$$

It may readily be checked that the general expression (4.5) does indeed satisfy the p.d.e. (4.1). In fact, it is even more readily checked that $1 / y^{2}$ (i.e. for $\Gamma=1$ ) satisfies it. More directly, $\sigma_{1}=\sigma_{0} \mathrm{C}^{2} / \mathrm{y}_{1}^{2}$ is an invariant (but unphysical) density: the corresponding $\sigma_{2}=\sigma_{0} \mathrm{C}^{2} / \mathrm{y}_{2}^{2}$ may be confirmed by inspection using (2.8) with (2.7b).

In general, if $\sigma_{1}\left(\mathrm{x}_{1}, \mathrm{y}_{1}\right)=\left(1 / \mathrm{y}_{1}^{2}\right) \Gamma\left(\Xi\left(\mathrm{x}_{1}, \mathrm{y}_{1}\right)\right)$, then the invariance of this density, i.e. $\sigma_{2}\left(\mathrm{x}_{2}, \mathrm{y}_{2}\right)=\sigma_{1}\left(\mathrm{x}_{2}, \mathrm{y}_{2}\right)$, may be confirmed directly by use of (2.8) with (4.7) and (2.7). It is now demonstrated that all such invariant densities are unphysical. Consider points within or on the circle which approach the boundary points $(x= \pm C, y=0)$ along the circular arcs $x^{2}+y^{2}=C^{2}-\alpha C y$, for $\alpha \geq 0, y>0$ and $\alpha \leq 0, y<0$. Then along these arcs, $g(x, y)=\left(1 / y^{2}\right) \Gamma(\alpha)$. For physical membrane densities, this must remain finite as $y \rightarrow 0$, so 


$$
\Gamma(\alpha)=0, \text { all } \alpha,
$$

i.e. $\Gamma$ is the zero function. Thus there are no physical self-isospectral (invariant) circular membranes in this scheme.

\section{Examples}

(i) Constant Density. The case of circular membranes isospectral with a uniform membrane, i.e. having the standard vibration spectrum with eigenfrequencies given by the zeros of ordinary integer-order Bessel functions of the first kind for fixed rim, or zeros of the derivatives of the Bessel functions for free boundary, was first presented in [1], for unit radius.

The membranes given by

$$
\sigma_{2}\left(x_{2}, y_{2}\right)=\frac{\left(1-a_{1}^{2}\right)^{2}}{\left[D_{a_{1}}\left(x_{2}, y_{2}\right)\right]^{2}} \sigma_{0}
$$

with $(2.7 \mathrm{c})$ for general radius $\mathrm{C}$, and $\sigma_{0}=$ constant, are isospectral with the uniform circular membrane of radius $C$, density $\sigma_{0}$. They are not only inhomogeneous but are evidently non-radial in density.

(ii) Density a Function of Radius. The case of membranes isospectral to radial-density membranes is of interest since inhomogeneous membranes with purely radial density functions are most commonly investigated (semi-)analytically or numerically (e.g. [6] [11]). The membranes isospectral to these in the scheme of this paper will no longer have purely radial densities, essentially because of the forms of (2.7). Indeed, it follows from the cancelled form of (4.8), viz.

$$
\left(c^{2}-r_{1}^{2}\right)=\frac{\left(1-a_{1}^{2}\right)\left(c^{2}-r_{2}^{2}\right)}{D_{a_{1}}\left(x_{2}, y_{2}\right)}
$$

together with $(2.7 \mathrm{c})$ in the form

$$
D_{a_{1}}\left(x_{2}, y_{2}\right)=1+\frac{a_{1}^{2}}{c^{2}} r_{2}^{2}-2 \frac{a_{1}}{c} x_{2}
$$

that

$$
\frac{r_{1}^{2}}{c^{2}}=\frac{a_{1}^{2}+\frac{r_{2}^{2}}{c^{2}}-2 a_{1} \frac{x_{2}}{c}}{1+a_{1}^{2} \frac{r_{2}^{2}}{c^{2}}-2 a_{1} \frac{x_{2}}{c}}
$$


which shows the non-radial nature explicitly. The isospectral densities are consequently angle-dependent and of the form $\sigma_{2}\left(\mathrm{r}_{2} ; \mathrm{x}_{2}\right)$.

In [12] it was shown that circular membranes with positive power radial densities of the form

$$
\sigma(r)=p^{2}(r / C)^{(2 p-2)} \sigma_{0}, p>1
$$

all have the radial part of their spectrum the same as for the homogeneous circular membrane (for the same radius $\mathrm{C}$ ) with constant density $\sigma_{0}$, for Dirichlet and Neumann boundary conditions. Then the corresponding non-radial-density isospectral circular membranes constructed as in the present paper are completely isospectral to the radialdensity membranes with (5.5), separately for each $\mathrm{p}$, but they all have the same radial spectrum as a uniform circular membrane of the same radius with constant density $\sigma_{0}$.

Perhaps the simplest explicit example has $\mathrm{p}=2$, i.e.

$$
\sigma_{1}\left(\mathrm{x}_{1}, \mathrm{y}_{1}\right)=4\left(\mathrm{x}_{1}^{2}+\mathrm{y}_{1}^{2}\right) \sigma_{0} / \mathrm{C}^{2} \equiv 4\left(\mathrm{r}_{1}^{2} / \mathrm{C}^{2}\right) \sigma_{0}
$$

and will be detailed here. From (2.8) with (5.3), and (5.6) with (5.4), one obtains

$$
\sigma_{2}\left(x_{2}, y_{2}\right)=4 \sigma_{0}\left(1-a_{1}{ }^{2}\right)^{2} \frac{\left(a_{1}^{2}+\frac{r_{2}^{2}}{c^{2}}-2 a_{1} \frac{x_{2}}{c}\right)^{3}}{\left(1+a_{1}^{2} \frac{r_{2}^{2}}{c^{2}}-2 a_{1} \frac{x_{2}}{c}\right)^{3}}
$$

as being completely isospectral with (5.6) (and radially isospectral with $\sigma_{0}$ ). 


\section{Discussion}

Compared with the one-dimensional case, not a lot of work has been done on inverse problems for two-dimensional continuous systems. (See e.g. [13] for a recent review. [14] is another earlier paper. [15] is a current related contribution in a more formal setting.) A consideration of which and how many spectral data should be considered for a possible unique reconstruction of the density function of a membrane (of a given shape) would be informed by the classes of isospectral membranes know to have identical spectra for certain boundary conditions but different densities.

The cases in this paper, of circular nonuniform membranes, with Dirichlet and Neumann boundary conditions, may yield some guidelines in this respect. For example, more than merely radial spectra of circles are required. One, or even both, complete Dirichlet or Neumann spectra are not even sufficient, at least for circular membranes.

This paper therefore leaves open the question as to whether some other additional spectra would be sufficient for a unique reconstruction procedure for a general membrane, and if so, what the other boundary conditions might be.

\section{Acknowledgements}

I thank Dr Maeve McCarthy for rekindling my interest in this problem. 


\section{References}

[1] Gottlieb H P W 1988 Density distribution for isospectral circular membranes SIAM J. Appl. Math. 48 948-51

[2] Saff E B and Snider A D 1976 Fundamentals of Complex Analysis for Mathematics, Science, and Engineering (Englewood Cliffs: Prentice-Hall)

[3] Gottlieb H P W 2002 Isospectral strings Inverse Problems 18 971-8

[4] Hamermesh M 1989 Group Theory and its Application to Physical Problems (New York: Dover)

[5] Jeffrey A 1995 Handbook of Mathematical Formulas and Integrals (London: Academic Press)

[6] Kalotas T M and Lee A R 1993 The modes of a circular membrane of non-uniform radial density Acustica $\mathbf{7 8} 220-5$

[7] Gutierrez R H, Laura P A A, Bambill D V, Jederlinic V A and Hodges D H 1998 Axisymmetric vibrations of solid circular and annular membranes with continuously varying density J. Sound \& Vibn 212 611-22

[8] Buchanan G R and Peddieson J 1999 Vibration of circular, annular membranes with variable density J. Sound \& Vibn 226 379-82

[9] Wang C Y 1999 Fundamental frequency of a wavy non-homogeneous circular membrane J. Sound \& Vibn 227 682-4

[10] Subrahmanyam P Bala and Sujith R I 2001 Exact solutions for axisymmetric vibrations of solid circular and annular membranes with continuously varying density $J$. Sound \& Vibn 248 371-8

[11] Willatzen M 2002 Exact power series solutions for axisymmetric vibrations of circular and annular membranes with continuously varying density in the general case $J$. Sound \& Vibn 258 981-6

[12] Gottlieb H P W 1992 Axisymmetric isospectral annular plates and membranes IMA J. Appl. Math. 49 185-92

[13] Gladwell G M L 1996 Inverse problems in vibration - II Appl. Mech. Rev. 49 S25S34

[14] Shen C-L 1993 On the nodal sets of the eigenfunctions of certain homogeneous and nonhomogeneous membranes SIAM J. Math. Anal. 24 1277-82

[15] Sun Z and Uhlmann G 2003 Anisotropic inverse problems in two dimensions Inverse Problems 19 1001-10 